\title{
Correction to: Intervendor variability of carotid intima-media thickness measurement: validation study using newly developed ultrasound phantom
}

\author{
Tomoko Ishizu $^{1} \cdot$ Hirotoshi Hamaguchi ${ }^{2} \cdot$ Naotaka Nitta $^{3} \cdot$ Yoshihiro Seo $^{4} \cdot$ Hiroshi Matsuo $^{5} \cdot$ Tsuyoshi Shiina $^{6}$
}

Published online: 1 July 2020

(c) The Japan Society of Ultrasonics in Medicine 2020

\section{Correction to: \\ Journal of Medical Ultrasonics (2020) 47:155-165 \\ https://doi.org/10.1007/s10396-019-00995-7}

In the Original publication of the article, Table 3 was published incorrectly. The correct Table 3 is given in this Correction.

Publisher's Note Springer Nature remains neutral with regard to jurisdictional claims in published maps and institutional affiliations.

The original article can be found online at https://doi.org/10.1007/ s10396-019-00995-7.

Tsuyoshi Shiina

shiina.tsuyoshi.6w@kyoto-u.ac.jp

1 Department of Clinical Laboratory Medicine, Faculty of Medicine, University of Tsukuba, 1-1-1 Tennodai, Tsukuba, Ibaraki 305-0817, Japan

2 Department of Neurology, Kita-Harima Medical Center, 926-253 Ichiba-cho, Ono, Hyogo 675-1392, Japan

3 Health Research Institute, National Institute of Advanced Industrial Science and Technology (AIST), 1-2-1 Namiki, Tsukuba, Ibaraki 305-8564, Japan

4 Department of Cardiology, Nagoya City University, 1 Kawasumi, Mizuho-cho, Mizuho-ku, Nagoya 467-8601, Japan

5 Matsuo Medical Clinic, 2-15-25 Kita-Honmachi, Yao City, Osaka 581-0802, Japan

6 Department of Human Health Sciences, Graduate School of Medicine, Kyoto University, 53 Kawahara-cho, Shogoin, Sakyo-ku, Kyoto 606-8507, Japan 
Table 3 Vendor participating in the study with the version of equipment provided and exported 2D image information

\begin{tabular}{llllll}
\hline Vendor & Ultrasound machine & Probe & Frequency bands & $\begin{array}{l}\text { Exported 2D image } \\
\text { resolution }{ }^{\mathrm{a}} \text {, mm/pixel }\end{array}$ & $\begin{array}{l}\text { Number of the pixel in } \\
\text { 10-mm length of IMT }\end{array}$ \\
\hline Fuji-Film & FC1 & HFL38 & $13-6 \mathrm{MHz}$ & 0.063 & 159 \\
Fukuda Denshi & UF-760AG+ & FUT-LA385-12P & $12-5 \mathrm{MHz}$ & 0.056 & 179 \\
GE & LOGIQ S8 & ML-15-D & $12 \mathrm{MHz}$ & 0.038 & 263 \\
Hitachi & ARIETTA 70 & L441 & $11-2 \mathrm{MHz}$ & 0.052 & 192 \\
Konica Minolta & SONIMAGE HS1 & L18-4 & $18-4 \mathrm{MHz}$ & 0.057 & 175 \\
Philips & EPIC7 & L12-3 & $12-3 \mathrm{MHz}$ & 0.046 & 217 \\
Siemens & ACUSON S2000 & 14L5 & $11 \mathrm{MHz}$ & 0.044 & 227 \\
Canon & Aplio i900 & 11L4 PLU-704BT & $11-4.8 \mathrm{MHz}$ & 0.060 & 167
\end{tabular}

${ }^{a}$ Exported 2D image resolution was presented as the tissue length/pixels of the picture from the depth set to $3.0 \mathrm{~cm}$ in each ultrasound machine preset for the carotid ultrasonography 